\section{Ageing defences}

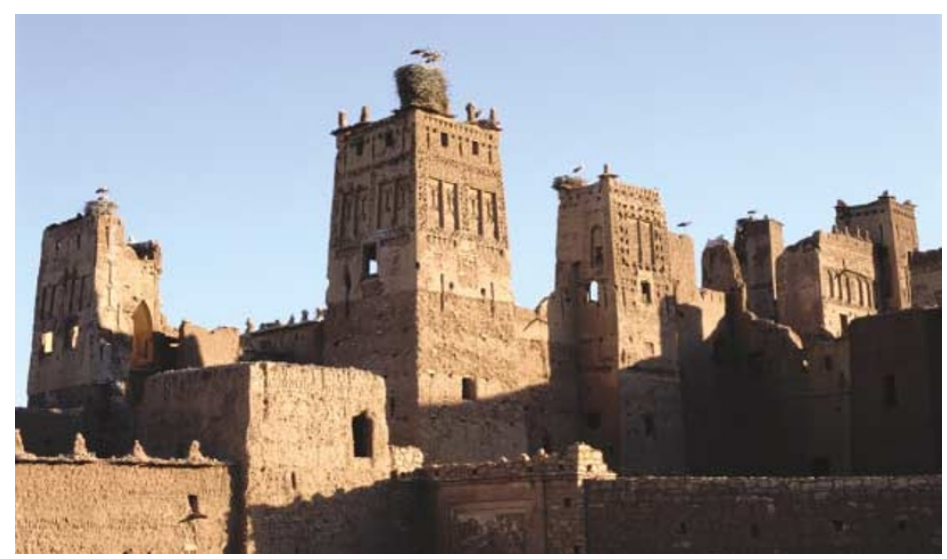

Those 'morning after feelings' get worse as you get older - ageing certainly has its problems, but the body can protect against some of these. Richard Van Etten and colleagues show that the antioxidant peroxiredoxin $1(\operatorname{Prdx} 1)$ is an important defence, which protects ageing mice from the harmful effects of oxidants.

The authors generated $\operatorname{Prdx} 1$-null mice, which were viable and fertile but had significantly shortened survival compared with wild-type littermates. Premature death was caused by haemolytic anaemia and formation of cancers, including B and T lymphomas, histiocytic malignancies and epithelial and mesenchymal tumours. These diseases were increased in heterozygotes and Prdx1 was found in most normal tissues, but was low or undetectable in tumours. So, Prdx1 seems to act as a tumour suppressor. Examinations in heterozygotes did not reveal structural rearrangement or loss of the remaining $\operatorname{Prdx} 1$ allele, indicating that mutations or epigenetic mechanisms cause the loss of $\operatorname{Prdx} 1$ expression. But what mechanisms cause tumour formation in the $\operatorname{Prd} x 1^{--}$mice?

$\operatorname{Prdx} 1^{-1-}$ mouse embryonic fibroblasts (MEFs) in vitro proliferated more slowly, were more sensitive to oxidants and had greater concentrations of peroxide-induced cellular reactive oxygen species (ROS) than wild-type MEFs. In addition, $\operatorname{Pr} d x 1^{1^{--}}$MEFs had higher basal and peroxide-induced concentrations of 8-oxoguanine - an oxidative DNA lesion that causes mutations. Collectively, this indicates that loss of Prdxl increases susceptibility to cancer by increasing sensitivity to oxidants, cellular ROS and oxidative DNA damage. Prdx1 has also been identified as an enhancer of natural killer (NK)-cell activity and NK cells are important for protecting against tumorigenesis. So, the authors examined NK cells isolated from $\operatorname{Prdx1^{-1-}}$ mice and found decreased lytic activity towards the murine NKsensitive T-lymphoma cell line YAC-1. Erythrocytes stimulate the cytotoxic activity of NK cells, but $\operatorname{Prdx1^{-/-}}$ erythrocytes were not as effective as wild-type erythrocytes at enhancing cytotoxic activity of wildtype NK cells. Conversely, wild-type erythrocytes had little stimulatory activity on $\mathrm{Prdx} 1^{-1-}$ NK cells, indicating that Prdx1 is required in both NK cells and non-immune cells for optimal NK function.

So, $\operatorname{Prdx} 1$ is directly implicated in tumour suppression in ageing mice. This work is a first step towards identifying the mechanisms that predispose $\operatorname{Prd} x 1^{-1-}$ mice to cancer and understanding how antioxidant pathways protect against tumour formation.

Emma Croager

\section{(2) References and links} ORIGINAL RESEARCH PAPER Neumann, C. A. et al. Essential role for the peroxiredoxin Prdx1 in erythrocyte antioxidant defence and tumour suppression. Nature 424, 561-565 (2003) WEB SITE

Richard Van Etten's lab:

Richard Van Etten's lab:
http://cbr.med.harvard.edu/investigators/vanetten /vanetten.html

\section{TRIAL WATCH}

\section{Ovulation and protection}

A vaccine against human papilloma virus 16 (HPV16) - which is the most common type of HPV and accounts for most cases of cervical cancer - has previously been shown to protect against HPV16 infection in women. However, most of the women in these studies were taking oral contraceptives, which regulate menstruation and production of sex hormones.

Nardelli-Haefliger and colleagues report in the Journal of the National Cancer Institute that women not taking oral contraceptives produce less antibodies to HPV16 after immunization and during ovulation than women taking contraceptives.

Seven women who were taking oral contraceptives and 11 women who were not were immunized with a recombinant HPV16 viruslike particle (HPV16L1 VLP) vaccine. All women developed detectable titres of anti-HPV16L1 VLP immunoglobulins (Ig) in their cervical secretions after immunizations. These titres were fairly constant in the contraceptive group throughout the menstrual cycle, but titres of both vaccine-specific and total IgG decreased by about ninefold during ovulation in the no contraceptive group, then rose again in the luteal phase of the cycle. The authors suggest that sex hormones might have a role in regulating antibody concentration in the cervix, and that the decrease in antibody concentration at ovulation might be a protective mechanism to reduce the level of antisperm antibodies in the genital tract at a time when conception is most likely. It is unclear whether the decrease in antibody production seen during ovulation will affect the ability of this vaccine to protect against cervical cancer.

ORIGiNAL Reference Nardelli-Haefliger, D. et al. Specific antibody levels at the cervix during the menstrual cycle of women vaccinated with human papillomavirus 16 virus-like particles. J. Nat/ Cancer Inst. 95, 1128-1137 (2003)

\section{HRT and a million women}

The Million Women Study was set up in the UK to investigate the relation between use of hormone-replacement therapy (HRT) and breast cancer incidence and mortality. The results from the study, published in The Lancet, confirm the findings of previous studies - use of HRT increases the risk of developing breast cancer. However, this study also shows that use of HRT increases the risk of death from breast cancer, and that the effect is greater with oestrogen-progestogen combinations than with other types of HRT.

1,084,110 women aged 50-64 were recruited between 1996 and 2001 and asked to fill in questionnaires about their use of HRT and their menopausal status. Over $50 \%$ of the participants had used HRT at some time. 9364 invasive breast cancers and 637 deaths from breast cancer were recorded. Users of HRT at recruitment were more likely than never users to develop breast cancer (relative risk (RR) 1.66) and die from it (RR 1.22). The risk began to increase within 1-2 years of starting HRT. Interestingly, past users were not at increased risk of developing or dying from breast cancer.

Women on oestrogen-progestogen HRT had a greater risk (RR 2) than oestrogen only HRT users (RR1.3). Use of HRT for 10 years is estimated to result in 19 extra breast cancers per 1,000 users of the oestrogen-progestogen combination.

ORIGINAL REFERENCE Million Women Study Collaborators. Breast cancer and hormonereplacement therapy in the Million Women Study. Lancet 362, 419-427 (2003) 